\title{
Caso productivo en una celda de manufactura flexible
}

\author{
Productive case in a flexible manufacturing cell
}

\author{
María Elena Bernal Loaiza ${ }^{1}$, Julián Alejandro Piedrahita Monroy ${ }^{1}$, Carlos Andrés Rivas Oyuela ${ }^{1}$ \\ ${ }^{I}$ Facultad de Ingeniería Industrial, Universidad Tecnológica de Pereira, Pereira Colombia. \\ mbernaldutp.edu.co
}

\begin{abstract}
Resumen- Este artículo presenta el diseño de un caso productivo que vincula los laboratorios virtuales de la Facultad de Ingeniería Industrial de la Universidad Tecnológica de Pereira, es decir, la celda de manufactura flexible (FMC) y el laboratorio móvil de logística, éste caso se denominó como el caso de producción de "Cuatrimotos PR". Éste se estructuró en detalle, procurando asemejarlo al proceso real de producción de cuatrimotos con su proceso logístico. Se describe la forma en que se diseñaron adaptaciones a los recursos existentes dentro de la celda de manufactura con el objetivo de ejecutar el proceso en el laboratorio.
\end{abstract}

Palabras clave - Brazo robot, FMC, Logística, Manufactura Flexible, Producción.

Abstract - This article presents a designed productive case which includes the virtual laboratories of the Universidad Tecnologica Pereira's Industrial Faculty, it means the flexible manufacturing cell and the mobile logistics laboratory; this case was named "PR ATV's production case". It was structured in trying to imitate a real process of production of ATVs with their logistics process. There is described the form in which is designed the adaptations of existing resources within the manufacturing cell with the aim of to execute the process in the laboratory.

Key Word - Robot arm, FMC, logistics, flexible manufacturing, production.

\section{INTRODUCCIÓN}

En la Facultad de Ingeniería Industrial de la Universidad Tecnológica de Pereira, se encuentran los laboratorios académicos denominados, Celda de Manufactura Flexible y Laboratorio Móvil de Logística, estos laboratorios han permitido a los alumnos de la facultad acercarse a los temas de manufactura flexible y logística de una mejor manera, esto principalmente se ha logrado a través de la labor desarrollada por el equipo de trabajo de estos dos laboratorios y por estudiantes que han desarrollado sus trabajos de grado utilizando estos recursos.
Es así como surge la posibilidad de utilizar los recursos de la FMC (celda de manufactura flexible) de la facultad con el objetivo de crear un caso productivo que permita el uso de este laboratorio aproximándolo a desarrollar procesos que se asemejen lo mejor posible a la realidad. Este artículo presenta un caso diseñado en el trabajo de grado llamado "Aplicación de Redes de Petri en los laboratorios académicos de la Facultad de Ingeniería Industrial", por lo que muestra parte de los resultados obtenidos con esta investigación, principalmente menciona el caso productivo "Caso de cuatrimotos PR " adaptado a la FMC de la Facultad de Ingeniería Industrial de la Universidad Tecnológica de Pereira.

El desarrollo de este caso productivo requirió del reconocimiento de los laboratorios académicos de la facultad por parte de los autores, aprendiendo además sobre su funcionamiento y adquiriendo experiencia en el manejo de los diferentes equipos con el motivo de distinguir sus alcances y la posibilidad de desarrollar procesos productivos en estos.

Se analizaron varios procesos productivos [1], buscando en la web la manera en que estos se desarrollan y priorizando sobre los productos que se fabrican con automatización, se comparó este método de fabricación con los alcances que permite la FMC de la facultad y se tomó la decisión de adoptar el caso productivo enunciado en este documento.

Este caso productivo es clave para que los estudiantes se motiven a aprender, esta motivación implica el gusto por conocer y reforzar nuevos conceptos; lo que induce a llevar a la práctica una acción [6].

\section{CONTENIDO}

A. Componentes laboratorio fmc [2] 
Los componentes de la FMC de la facultad de ingeniería industrial de la Universidad Tecnológica de Pereira son los siguientes:

\section{Estación Manager.}

Es el computador principal donde están los diferentes aplicativos para manejar la celda en su conjunto, o para operar de forma independiente componentes como el robot, el almacén o la cinta, permitiendo planear la producción y dar la orden de fabricación, así mismo se puede simular el proceso en 3D. La estación manager tiene instalado el software COSIROP para controlar el brazo robot, el software MINITEK IV para controlar el funcionamiento de la FMC en conjunto y además el PCTV Visión para controlar la cámara de la inspección de calidad; este computador además tiene dos tarjetas tipo PSI para la transferencia de datos.

2. Almacén de Materia Prima y Producto Terminado:Es un almacén automatizado que consta de 14 posiciones organizadas verticalmente en dos columnas con sensores inductivos que indican al sistema si la posición se encuentra libre o en uso. El almacén tiene un grupo de contenedores especiales para ubicar piezas cilíndricas y prismáticas construidas a partir de polímeros o aleaciones de aluminio por su facilidad para maquinar prototipos.

3. Pinza Robótica: Hace parte de la estación de almacén, ubica la materia prima y producto terminado. Se desplaza hacia las 14 posiciones del almacén para tomar el contenedor de la pieza indicada, llevarlo al pallet y viceversa, tiene un track o pista de posiciones que se mueve horizontalmente y la pinza se desplaza a través de este para alcanzar la posición requerida.

4. Cinta transportadora: Es un sistema de transporte rectangular a escala académica, con arrastre por cinta y accionado por motores de marca BONFIGLIOLI, la marcha de estos motores y el accionamiento de topes de detenimiento para los pallets son controlados desde el software MINITEK.

5. Pallet: Se utilizan para transportar los contenedores sobre la banda transportadora, son de material metálico, y presentan guías en su parte inferior para ser conducidos en la dirección de la banda transportadora, además para ser detenidos por los topes en una estación de trabajo determinada.
6. Robot Articulado Angular: Robot MITSUBISHI Melfa $\mathrm{RV}-2 \mathrm{AJ}$, es un manipulador de material liviano, tipo industrial con 5 grados de libertad 1 el cual se mueve a través de un eje de guiado lineal con 6 posiciones para diversas tareas. El principal uso de este robot es la distribución y alimentación de material a la banda transportadora, al centro de mecanizado y la estación de ensamble. [3]

7. Estación de Trabajo y Ensamble: Consiste de una mesa metálica ubicada en una de las esquinas de la FMC, cerca al robot articulado, permite la proximidad de éste (específicamente en su track \#3) para realizar operaciones de ensamble, simulación de corte, simulación de soldadura, entre otras más que se quieran realizar.

8. Centro de Mecanizado CNC. Fresadora: Fresadora ODISEA de Alecop, controlada mediante CNC Fagor 8025 o 8050/55. Es una máquina-herramienta utilizada para dar formas complejas a piezas de metal $\mathrm{u}$ otros materiales. Es una máquina que puede ejecutar una gran cantidad de operaciones de mecanizado complejas, como cortes de ranuras, superficies, perforaciones, encaminado, entre otras, en general la fresadora permite maquinar planos $y$ superficies sobre un material determinado.

\section{COMPONENTES LABORATORIO MÓVIL DE LOGÍSTICA}

Los componentes del laboratorio móvil de logística de la facultad de ingeniería industrial de la Universidad Tecnológica de Pereira son los siguientes:

A. Servidor: es el componente responsable por el procesamiento de la información en tiempo real, desde el ingreso de datos hasta la salida de informes de los mismos, sus características son similares a cualquier sistema operativo de Windows, habilitado para conexiones de red física e inalámbrica, con aplicativos para controlar el resto de sus componentes, como también para hacer la gestión de la información en tiempo real.

El servidor utiliza un router a través del cual genera su propia red inalámbrica lo que facilita que al conocer la IP y claves asignadas, bien sea a uno de los 2 reader existentes o la del servidor mismo, cualquier usuario puede desde cualquier punto dentro del radio de la señal hacer demostraciones prácticas del Laboratorio.

\footnotetext{
${ }^{1}$ Grados de libertad: corresponde principalmente a los ejes que dispone, lo que le permite mayor cantidad de movimientos y posiciones para adoptar, debido a que cada eje se puede mover independientemente.
} 
B. Impresora Datamax serie H 4212 X: Funciona a través de transferencia térmica fijando en la etiqueta las porciones de cinta que representan el código de barras, al igual que cuenta con un módulo para grabar los tags o microchips de la etiqueta donde se almacena la información del producto para la posterior lectura e intercambio de datos de manera electrónica (RFID), de los productos creados.

C. Antenas de diseño ThingMagic: son módulos ó dispositivos electrónicos de funcionamiento con la tecnología RFID ó de Radiofrecuencia.

Estos dispositivos están dispuestos a la entrada y salida del centro de distribución, para la recepción de producto y su respectivo despacho etiquetado con RFID, gracias a la polarización circular que las antenas realizan, garantizando la lectura en cualquier posición e incluso sin necesidad de estar extremadamente cerca de las mismas.

D. Readers: son la unidad central de procesamiento de la información enviada por las antenas que luego se envía al servidor, donde se encuentran los aplicativos necesarios para la gestión de la información de los inventarios en tiempo real.

E. Lectores de Códigos de barra, marca HandHeld: son pistolas para la lectura de los códigos de barras de los productos, empaques y cajas.

F. Góndola inteligente de almacenamiento: Consta de una estantería similar a la estantería de almacenamiento, tiene adaptada una antena y un reader, lo que permite detectar todos los productos que posean chips de radiofrecuencia, esta góndola permite llevar un control instantáneo y constante del inventario ubicado en ella.

\section{DESCRIPCIÓN DEL CASO}

El caso productivo plantea 8 estaciones de trabajo identificadas cada una con sus recursos humanos, físicos, herramientas, requerimientos de materias primas y las funciones que se asignan a cada estación. Se cuenta con operaciones humanas, y operaciones automatizadas posibles gracias al Robot.

Se requieren aproximadamente 7 personas, para la planta de producción de las CUATRIMOTOS, aunque de acuerdo a la toma de tiempos, el análisis de movimientos y el análisis de funciones, se puede reducir el número de personas, de acuerdo a la creatividad del organizador de la planta productiva.

Las herramientas a usar en el proceso son:
- Destornilladores para el chasis y las llantas

- Destornillador imantado para la carrocería de la moto

- Base para chasis

- Pallets

- Base para la carrocería

- Software Minitek y Cosirop

- Software logístico

- Góndola inteligente.

En cuanto a los tiempos, se puede experimentar y hacer prácticas de métodos y tiempos al caso planteado para posteriores desarrollos.

\section{ESTACIONES DE TRABAJO}

Para la definición de actividades se pensó en la parte de planeación y programación de la producción en la estación uno, la distribución de materias primas en la estación dos, el proceso productivo en las estaciones tres a la siete, y la parte logística en la última estación.

Primera Estación (Estación Manager):

1. Se define el plan de producción con las unidades de CUATRIMOTOS, que irán en el pedido, para el cliente final, bien sea por pronósticos, o por la demanda real del cliente final.

2. Luego de definido el plan de producción se presentan dos actividades concurrentes que son:

2.1 La programación en el MINITEK, del número de CUATRIMOTOS a producir, y el uso de los recursos de la Celda, activando así la celda.

2.2 Se activa el comienzo de actividades en la estación dos.

Segunda Estación (Distribución de Materias Primas e Insumos):

3. Se inicia actividades en la estación dos con la planeación de materiales y de recursos para cada una de las estaciones a las que hay que proveer.

4. Se realiza el control de materias primas, registrando la salida de los elementos con los que se proveerán las estaciones de la planta.

5. Un patinador distribuye los materiales ó insumos a cada una de las estaciones de trabajo, siguiendo la mejor política de distribución de materiales en almacén, llevando:

5.1 Dos Piezas de chasis naranja, 1 plataforma y 1 tornillo naranja a la $3^{\mathrm{a}}$ estación. 


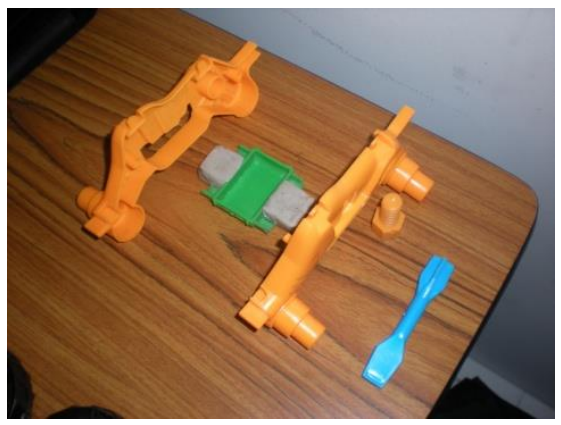

Figura 1: piezas y herramienta para la tercera estación.

5.2 Manubrio de la moto, 2 tornillos metálicos y frente de la moto a la estación 4.

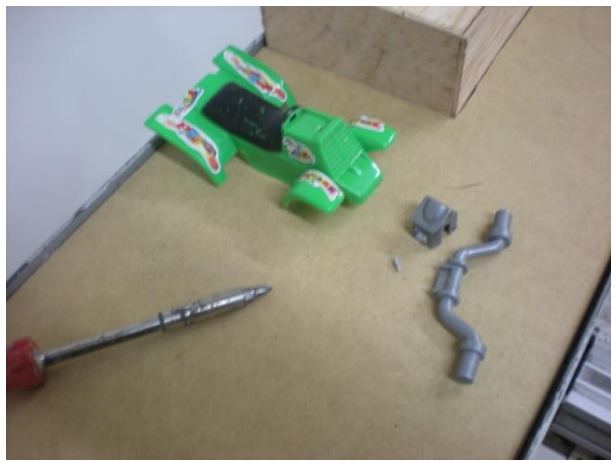

Figura 2: piezas y herramienta para cuarta y quinta estación.

5.3 Cuatro tornillos naranja, 4 llantas, acabados de motor, parachoques y parrilla a la $6^{a}$ estación.

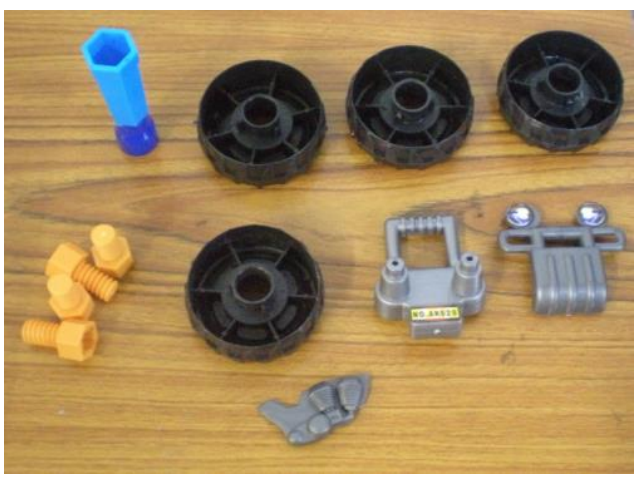

Figura 3: piezas y herramienta para sexta estación.

6. Ya activada la FMC (Actividad 2.1) la pinza se desplaza del almacén hacia la banda transportadora donde hay un pallet sostenido por topes en la salida del almacén, esperando para llevar la base para chasis a su destino.
7. Se bajan los topes de la banda transportadora y se lleva el pallet con la base para chasis, a la estación tres.

Tercera Estación (Subensamble de Chasis):

8. Se activan los topes en la estación tres, donde puede ocurrir qué:

8.1 El operario espere a que todo esté listo, ó se le asignen labores de alistamiento de la estación debido a que:

8.1.1 Llegaron las materias primas completas a la estación, pero no ha llegado el pallet con la base para chasis.

8.1.2 Llegó el pallet con la base para chasis, pero no han llegado las materias primas completas necesarias en la estación

8.2 El operario ensambla el chasis, y asegura el chasis con un tornillo naranja

9. Se desactivan los topes en la estación tres, y se transporta el pallet con el chasis a la estación 5 a través de la banda transportadora.

Cuarta y quinta estación (Subensamble de carrocería y ensamble de chasis y carrocería):

Antes de mencionar las actividades de esta estación se aclara que la carrocería por su tamaño no se patina, sino que llega a una zona dentro de la estación, directamente desde el momento en que ingresa a la planta, por lo que para este caso se supone que esta estación de trabajo posee en la planta suficiente carrocería.

10. Ya provistos los materiales de la estación 4 (actividad 5.2), se ensambla la carrocería.

11. Realizada la actividad 9, se activan los topes en la estación cinco, se detiene el pallet, y el robot toma de la base el chasis ensamblado y lo lleva a la mesa de trabajo donde lo ubica.

12. El Robot efectúa el ensamble de la carrocería sobre el chasis, y luego el operario de la estación asegura el ensamble con un tornillo.

13. El Robot lleva el producto procesado al pallet con la base, que están en la cinta transportadora.

14. El Robot termina la operación, se desactivan los topes y el pallet continúa por la banda transportadora para llegar a la $6^{a}$ estación.

Sexta estación (Ensamble de llantas y adicionales):

15. Se activan los topes en la estación 6, se detiene el pallet con el producto en proceso, donde puede ocurrir que:

15.1 No se pueda efectuar el ensamble de las llantas, acabados de motor, parachoques y parrilla, teniendo el operario que esperar debido a que:

15.1.1 No se poseen los insumos completos necesarios para realizar la operación, aunque el pallet esté con el producto en proceso. 
15.1.2 Se poseen los insumos pero no ha llegado el ensamble de la $5^{\text {a }}$ estación.

15.2Se efectúe el ensamble de las llantas, acabados de motor, parachoques y parrilla ya que se poseen con todos los recursos necesarios, estando el producto listo para las pruebas de Calidad.

Séptima estación (Aseguramiento de la Calidad):

Calidad: Basada en el punto de vista de que los productos y los servicios deben cumplir con los requerimientos de quienes lo usan [7].

16. En la estación de calidad puede ocurrir que al realizar las pruebas, con los recursos disponibles:

16.1El producto esté defectuoso, por lo que se harán correcciones al producto, y se volverán a hacer las pruebas de Calidad.

16.2El producto esté conforme, por lo que pasará al área de logística.

Hay muchas maneras de definir la calidad

\section{Octava estación (Logística):}

17. Se programa y configura en el Servidor el tipo de etiqueta ó código que manejarán las CUATRIMOTOS con etiqueta RFID, guardando este en la base de datos.

18. En la Impresora se imprime el código o número asignado anteriormente.

19. Se pega el código impreso RFID en las CUATRIMOTOS.

Para actividades propias de almacenamiento antes de la distribución final, la cual es una política de inventarios de la empresa productora de CUATRIMOTOS, tenemos que:

20. Se lleva a la zona de almacenamiento o zonas de estanterías, se lee la etiqueta con las antenas y el reader RFID ubicados en la góndola.

21. Se registra automáticamente, en el servidor, el número de inventario, de producto que se haya identificado en el tag RFID.

Después de almacenado el producto, de acuerdo a los pedidos de despacho, y las políticas de inventarios, se continúa el proceso así.

22. Se retira las CUATRIMOTOS que se han de despachar previa solicitud del cliente. Se lee la etiqueta con las Antenas y el Reader RFID en la góndola inteligente, cuando esta se retira.

23. Se registra en el servidor, automáticamente las descripciones del o de los producto (s) que sale(n) del almacén.

Se despacha la mercancía desde el Centro Logístico al Cliente que solicitó la mercancía, por el medio que se considere más pertinente.

\section{ADAPTACIÓN DE LA CELDA DE MANUFACTURA PARA EL PROCESO DE ENSAMBLE}

Las actividades desarrolladas para adaptar todos los componentes necesarios para el proceso de ensamble se pueden dividir en varias etapas, como sigue:

- Elaboración de bases.

- Elaboración de topes.

- Elaboración de bases para mesa de ensamble.

\section{A. Bases}

Se requería elaborar bases que se adaptaran a los contenedores y que permitieran que el chasis de cuatrimoto se adaptara con mucha exactitud, de tal manera que al momento de realizar todas las operaciones en éste no se presentaran inconvenientes, principalmente referentes a movimientos de las piezas y diferentes posiciones de acuerdo a la ubicación dada por el operario.

Todo esto debido a que como segunda estación de ensamble se encuentra la ejecución de algunas operaciones del robot lo que hace muy necesario que haya precisión para que este siempre tome las piezas en la misma posición con que se programó anteriormente.

Se elaboraron dos tipos de bases que cumplen con la misma función, un tipo fue desarrollado con porcelanicrón y masilla epóxica, el otro tipo de base se elaboró con resina r-740 combinada con endurecedor 744 y vaciada en un molde para obtener la base que en últimas fue pulida y terminada con masilla epóxica.

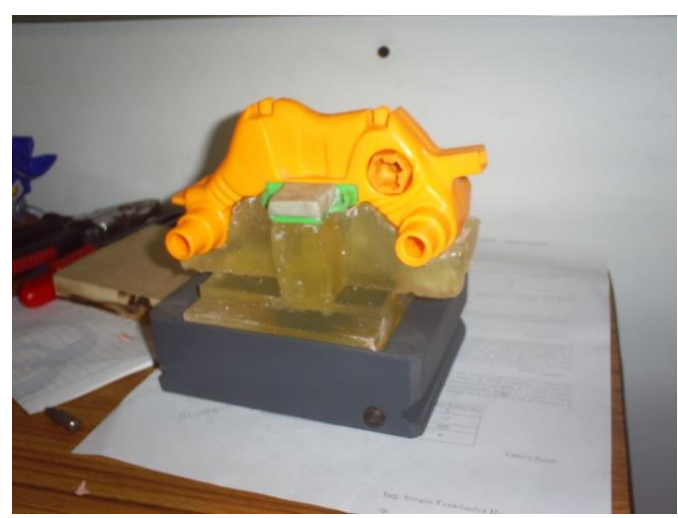

Figura 4: base para chasis

\section{B. Topes}

Con el objeto de elaborar u obtener topes que pudiesen detener el pallet en cada estación de trabajo montada dentro del recorrido de la banda transportadora para el desarrollo de este proyecto, se adquirieron dos fallebas de incrustar, modificándolas para obtener el diseño finalmente observado en 
la siguiente figura, estas se instalaron de tal manera que pudiesen ser removidas y que permitieran la activación y desactivación del tope en el momento deseado por el personal de las estaciones 3 y 6 .

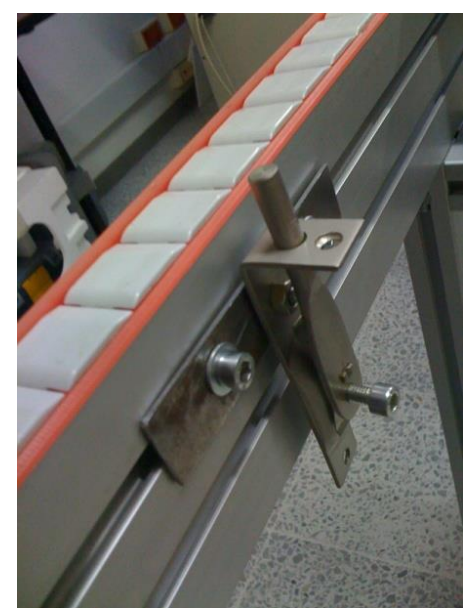

Figura 5: tope ubicado en banda transportadora.

C. Bases en mesa de ensamble

En el momento que se estaba programando el robot para realizar el proceso de ensamble de la carrocería sobre el chasis, se descubrió que el robot no tenía buena posibilidad de movimiento para este proceso por lo tanto se recurrió a elaborar dos cajas de madera que permitieran que cada una de las bases, es decir la base para el chasis y la base para la carrocería quedaran lo suficientemente altas y aptas para que el robot pudiera manipular las piezas.

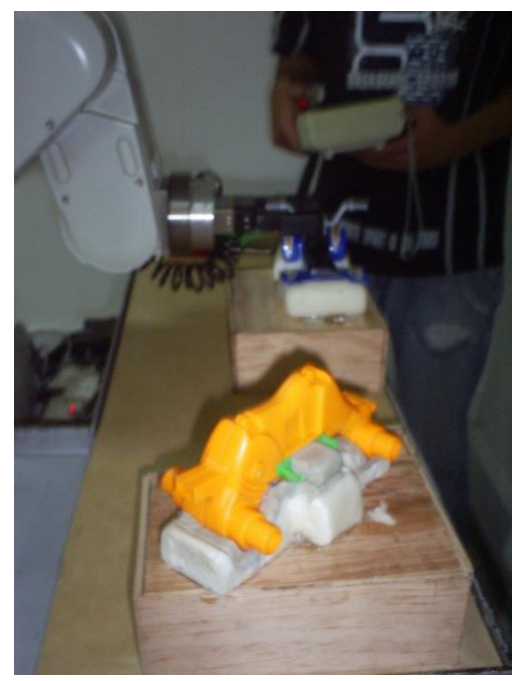

Figura 6: bases en mesa de ensamble.

\section{PROGRAMACIÓN}

Para el desarrollo del proceso de ensamble se debió programar el brazo robot utilizando el programa COSIROP y para la Celda de Manufactura el programa MINITEK.

\section{VII.PRÁCTICA}

La práctica se realiza con un grupo de estudiantes, a los cuales se les debe dar unas instrucciones sobre los materiales y herramientas necesarias para cada estación de trabajo, además el proceso que les corresponde ejecutar y se explica el objetivo de la práctica, resaltando los conceptos académicos que permite aplicar, de acuerdo a la asignatura que cursen los estudiantes visitantes.

Estos conceptos académicos deben tener un enfoque basado en competencias [5], de ahí la importancia de trabajar en laboratorios con tecnología moderna que propicien plantear diferentes simulaciones de casos productivos.

\section{CONCLUSIONES}

Diseñar el caso de producción dentro de los laboratorios de la facultad, conllevó a conocer muy bien la capacidad de los recursos disponibles, las ventajas de la celda de manufactura flexible y el laboratorio móvil de logística.

Adaptar un proceso productivo en un montaje o distribución ya establecido es complicado, por lo tanto se afirma que el mejor camino para establecer un proceso productivo utilizando un sistema de manufactura flexible se presenta cuando se distribuye o se monta los componentes o herramientas de la FMC con base al proceso productivo del producto a ensamblar o fabricar, en caso contrario se deben diseñar las adaptaciones necesarias al producto y al sistema, como en nuestro caso ocurrió con los topes de la banda transportadora, las bases para el chasis y la modificación a una pieza de la cuatrimoto.

El proceso de adaptación para fabricar o ensamblar un producto en un sistema de manufactura flexible se hace bastante complejo cuando este sistema ya ha sido montado y fijado, es decir, adaptar un proceso productivo en un montaje o distribución ya establecido es complicado, por lo tanto se afirma que el mejor camino para establecer un proceso productivo utilizando un sistema de manufactura flexible se presenta cuando se distribuye o se monta los componentes o herramientas de la FMC con base al proceso productivo del producto a ensamblar o fabricar.

La simulación de un caso productivo induce a los estudiantes a que analicen la posibilidad de adaptación que permiten este tipo de celdas, además invitarlos a diseñar nuevos productos y procesos que se implementen en esta línea de producción. 
La práctica de laboratorio permite involucrar activamente al estudiante en crear y construir modelos mentales robustos. Los robots en la educación crean ambientes donde los estudiantes pueden experimentar y reexperimentar el concepto en varios contextos [4]. Después de ejecutar la práctica se observó que muchos estudiantes obtienen conclusiones destacables y observaciones referentes a cambios en las estaciones de trabajo.

\section{REFERENCIAS}

[1] Procesos industriales de manufactura. [Online] Disponible en: http://robotico15.blogspot.es/1227210240/procesosindustriales-de-manufactura/

[2] Manual Técnico Célula Minitek VI, Laboratorio de Manufactura Flexible: Universidad Tecnológica de Pereira.

[3] MITSUBISHI. Mitsubishi Industrial Robot CR1/CR2/CR3/CR4/CR7/CR8 Controller Instruction Manual. Detailed explanations of functions and operations, Universidad Tecnológica de Pereira

[4] A. Delgado, Inteligencia Artificial y Minirobots. Bogota: Ecoe, 1998, p. 213

[5] J. H. Pimienta, Estrategias de enseñanza-aprendizaje, 3er ed. México: Pearson, 2012, p. 101

[6] F. Díaz y G. Hernández, Estrategias docentes para un aprendizaje significativo, 3er ed. México: Mc Graw Hill, p. 53.

[7] D. Montgomery, Control estadístico de la calidad, 3er ed. México: Limusa-Wiley, 2004, p. 4. 